\title{
Using Gamification to motivate occupants to energy efficiency in a social setting of a Building automation system
}

\author{
Joana Páris, João Cambeiro, Vasco Amaral, Armanda Rodrigues \\ Departamento de Informtica \\ Faculdade de Ciências e Tecnologia \\ Universidade Nova de Lisboa \\ Lisbon, Portugal \\ \{jpa.pereira,jmc12976\}@ campus.fct.unl.pt and \{vma, a.rodrigues\}@ fct.unl.pt
}

\begin{abstract}
The widespread use of IoTs, as cheap and immersive technology, is enabling a wide range of daily life systems where Humans play a central role. It is commonly accepted that Humans sometimes present non-reliable behaviour. On the other hand, Gamification is becoming a common technique in system development to integrate business logic and induce Humans to accomplish certain goals and enforce systems reliability. Due to human nature, the evolution of game logic becomes an essential aspect of such systems to keep users engaged and participative.

In this paper, we illustrate and discuss evolution in the particular scenario of a running Office Automation System in our open space. Here, the Human plays different roles such as an actuator, source of system input, a controller (decision maker), or simply environment (Human-in-the-loop). The mentioned system is the result of partially retrofitting a room of a forty years old building. It runs with a dynamic context scenario (that motivates different setups) and is formed by a heterogeneous set of IoTs. Those types of equipment are integrated to mainly accomplish two, sometimes conflicting, main goals: energy efficiency and Human comfort.
\end{abstract}

As we will describe next, given the complexity of our system, various system's requirements need to be fulfilled at the same time. Those will dynamically change during runtime to contribute to both efficiency and participants' engagement. Not only the game requirements of the system evolve, but also the participants' behaviour change.

We have consulted the open space's occupants on their daily routines and their preferences towards Gamification and gamified systems, particularly considering their social settings. They were also consulted on their views towards achieving energy efficiency in the open space. The results of this assessment are presented in this paper. The major suggestions were integrated into the current design of the system. We considered those that could contribute to the system's efficiency and reliability according to the system's goals. Besides, we also considered those that led to the use of several game techniques for motivating and improving the Humans' participation.

Index Terms-Gamification, Evolution Requirement, Energy Efficiency, Building Automation Systems, CPS, Human-in-theLoop

\section{INTRODUCTION}

Concerns about sustainability have greatly increased in the last decades, as humans are becoming more aware of their impact and the changes in the planet's health [1]. Due to this

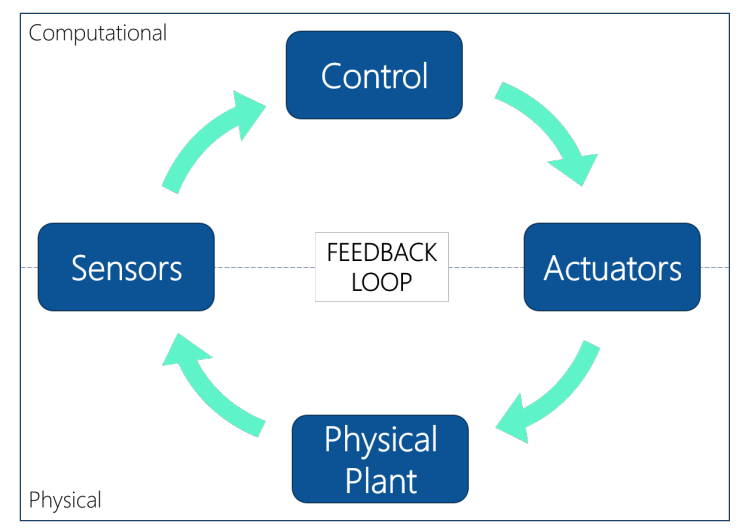

Fig. 1. CPS feedback loop, adapted from [4]

fact, many companies started to release devices that aim to manage resource consumption efficiently. Today, we can find intelligent buildings equipped with devices such as sensors, smart outlets or lights, that can control, monitor and report on daily activity. Those are also capable of establishing an autonomous functioning environment by communicating with each other. These types of settings, called cyber-physical systems (CPS), are programmed to trigger a response when a certain condition in the environment is noted [2]. In a continuous feedback-loop, once the Physical environment suffers some modification, that change is read by the sensor(s) and is reported to a control unit. According to the values read, this unit can trigger a certain action to be carried by an actuator, as depicted in Figure 1. Some of these CPS, particularly building automation systems (BAS) are capable of managing indoor conditions, like lights and heating [3].

However, in some cases (mostly in retrofitting scenarios) due to the infrastructure design and limitations, some features cannot be operated autonomously. In these contexts, the system will need the intervention of a human counterpart to be able to act. For example, opening a window to reduce the indoor temperature. CPS that incorporate humans are called Human-in-the-Loop CPS (HitLCPS) [5]. In the previous 
example, the Human provides the closing of the feedback loop. Unfortunately, due to human behaviour unpredictability, the system cannot fully rely on the expected participation. We thus propose to use gamification technology to answer these types of issues. Common game techniques will provide occupants with the needed motivation to interact in other contexts, and increase participation in various types of systems [6].

A gamified CPS system as we propose is subject to requirements evolution during its runtime [7], [8]. Not only in terms of functional goals but also in terms of the gamification logic itself. The reason is that, at some point, the humans' engagement stabilises and the need for gamification engagement becomes less evident.

We have instrumented and automated an open space with sensors and actuators to have energy savings without interfering with the occupant's comfort. This system is composed of a heterogeneous set of IoTs operating in an open source integration platform.

In this paper, we present the design of a proposed gamification strategy to integrate with the system. As will be discussed further on, this design is based on the input (user assessment) of the group of occupants of the open space.

In the following sections, we will discuss related work, present our case study and target audience. Afterwards, we will describe our game strategy and evolution, present the conclusions of the paper and the future phases of the project.

\section{RELATED WORK}

Several examples of systems that use Gamification to increase energy savings or user engagement have been identified in the context of this work.

In [9], a group of researchers developed a smart meeting room that detected the presence and regulated the room temperature and the state of the lights and projector. The goal was to decrease energy use and provide both a productive and comfortable space. The system was fully autonomous, making control decisions according to the reports. The main difference between this setup and our proposal is the human intervention in the control and tasks (acting as sensor and actuator), which brings a new set of requirements.

The platform described in [10] used a chat component to provide a way to elicit requirements for a system. Two versions were developed (with and without Gamification), to assess if Gamification improves engagement and performance. Performance of both settings is provided in the study. The results demonstrated that the group using the gamification version produced more and better material than the other group. We could also conclude that: the control group i) (without Gamification) had a stronger collaborative dynamic; whereas the treatment group ii) was more competitive among itself with negative impact in the relations between group members. However, we hypothesise that a different set of gamification elements would create a different outcome. In fact, we believe that a balance between collaboration/competition and extrinsic/intrinsic could have been achieved.
Energy chickens [11] is a game with a virtual chicken barn that intends to reduce the plug-load in a commercial office, where the chickens' health and the eggs they laid depended on the consumption measured. This was an experience that relied only on human behaviour and which was able to accomplish a decrease in energy use. However, once there was an optimised routine in place, the chickens would continue to lose health, which confused the players. Therefore, special care has to be taken in the gamification logic design to this evolution aspect. When the system reaches stability, leading the Human to the most efficient behaviour, the context changes (or evolves) and the Gamification logic also has to adapt to the new reality. If the gamification logic does not evolve, there is the risk of bringing more harm than good to the system, and unexpected human behaviour (or disinterest).

\section{The CAse STUdy}

Project Smartlab is an open space in the research Lab NOVA LINCS of the Department of Informatics at Faculdade de Ciencias e Tecnologia at Universidade Nova de Lisboa (Portugal). It is equipped with workstations and a variety of communicating devices in contact with the room control, as shown in Figure 2. The open space is thus a BAS CPS, a pioneer open space shared by both $\mathrm{MSc}$ and $\mathrm{PhD}$ students. It is located in a dated building, which was not originally designed to accommodate automation. A variety of devices, used for monitoring and control, transformed the space in a retrofitting environment, which is now capable of reducing energy consumption while offering a comfortable environment for its occupants [4] (Figure 3).
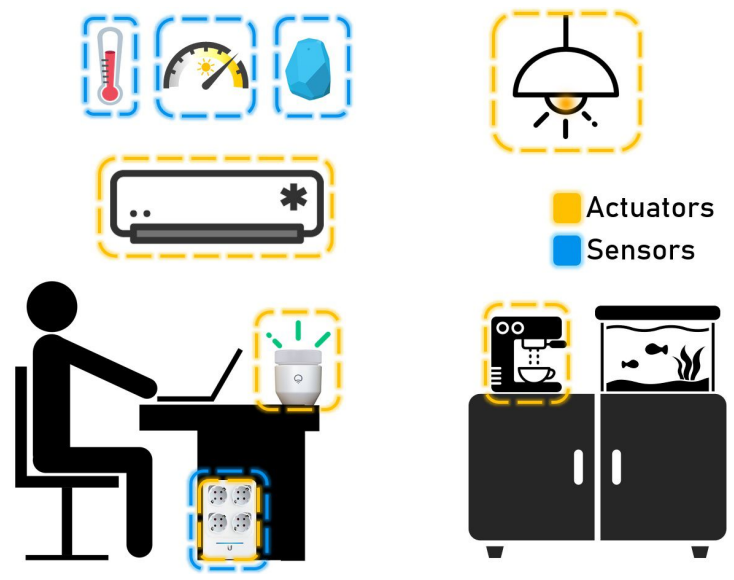

Fig. 2. Representation of the open space devices.

The space includes several sensors to monitor indoor and outdoor conditions: Estimote beacons ${ }^{1}$ to measure temperature and luminosity; Ubiquiti $^{2}$ power sockets to measure power and current values; energy meters for the air conditioner consumption; and outdoor thermometers and sensors to measure air temperature and visible light, respectively. The system

\footnotetext{
${ }^{1}$ https://estimote.com/

${ }^{2}$ https://www.ubnt.com/
} 
also contemplates actuators for changing different aspects of the room, such as the state of several components and the settings of some devices: Ubuquiti power sockets, Lif $\mathrm{x}^{3}$ lights, air conditioning, conventional halogen lights and a coffee machine can be automatically turned on and off. Moreover, the saturation, brightness and hue settings of Lifx lights, as well as temperature settings, can be altered. Additionally, the room features a warm water aquarium, a CPS system in itself, which is managed by the Open Aquarium hardware solution, to provide optimal conditions to its inhabitants [12].

Humans can play the role of sensor devices when directly asked by the system about their opinion on specific room conditions. They can also play the role of actuators if the system asks them to perform some task. They can also perform supervised control on the system, analysing the information provided by the system and deciding accordingly. This set of components allows the system to know the overall conditions of the room and, based on that data, trigger a specific action in response, resulting in a closed feedback loop [13].

These state changes directly affect both the occupants and the aquarium. They also influence the environment and therefore the control decisions. In the current setting, the system works towards efficiency, while providing optimal acclimatisation conditions. However, changes in the occupants' behaviour, if adequately motivated, may still lead to efficiency improvements. We propose that this can be achieved through the use of gamification techniques.

\section{A. Occupants' Assessment}

As mentioned before, this space is used by Computer Science postgraduate students while working on their projects. It should be a comfortable, relaxed, non-disruptive environment where the occupants can focus on their work.

The students were asked to fill in a questionnaire to learn about their routines and preferences. This way we could ground the design of the game system according to their needs. Six responses to the questionnaire were gathered in total, corresponding to all the room's occupants. The survey included basic questions to categorise the population, and additional questions about their knowledge and opinions on Gamification and gamified systems, with a focus on the social issues of the open space. The students were also questioned on their preferences in gaming features in the setting. The questionnaire ended with questions on energy efficiency. Below, the results of the poll are described.

Half of the population in the smartlab works full-time on their doctoral or master thesis and spend most of their time in the office, between two and a half to six days a week. Their home is usually their second choice for working. The rest either worked part-time elsewhere or had already finished their thesis.

Regarding improvements to the space conditions, students mentioned comfortable office furniture, better climate control and accessible switches, and a revision of the network circuits.

\footnotetext{
${ }^{3}$ https://www.lifx.com/
}

In the context of collaboration in the workplace, the students were questioned regarding their opinions on group work and on exchanging ideas, and whether it was something they would typically be engaged. Overall, the responses were positive, the population sees the benefits in teamwork and in discussing their work with colleagues, although they would not do that every time. Only two individuals do not have this habit.

On gamification issues, $50 \%$ of the population enjoyed gamified systems and the rest either never had used them $(16.7 \%)$ or had no opinion on the matter. When asked about which characteristics they could identify in the customarily gamified systems, the standout aspect was social interaction, followed by avatar customisation, progress or statistics display and rewards. Teamwork, competition, ranking, missions and scores were present in some of the systems as well.

On the importance of the features used in gamified systems, the students preferred real-time performance feedback and the leaderboard, with half of the population votes, as shown in Figure 4. The ability to know how they are progressing helps them to quantify their efforts and motivates them to improve their actions. The leaderboards serve as a guide for them to understand where they are when compared to other players. Additionally, levelling, teamwork, challenges and rewards were also favourites. The least preferred were competition among users, customisation of avatars, achievements and social forums.

From the data gathered we concluded that the individuals highly value any feedback reporting and like to be challenged. They enjoy working as a team, which they find more stimulating and fun than working alone. However, when comparing their preferred elements and the ones they use we can observe that the offer does not fully meet the requests.

When questioned about energy efficiency, the entire population sees the importance of managing energy use, the individuals are aware of the problem and believe it is possible to achieve a functional planet-friendly open space by using a gamified system.

The answers to this questionnaire enabled us to know what our target audience does, needs and prefers. From the previous analysis, we concluded that our subjects are aware of the energy costs linked to intelligent environments and are receptive to the idea of using technology to reduce these expenses. We are working with a group that enjoys and acknowledges the benefits of group dynamics. The majority is familiarised with gamified systems, finding them appealing. In this scenario, awareness is essential, as the occupants like to have some reference to their status results in the game. This knowledge includes the individual results concerning the rest of the community, which is a stimulus for self-improvement. They also appreciate participating in missions, which gives them a social feature and a sense of growth within the game.

\section{GAME LOGIC}

The design of the game logic considered the occupants' assessment and energy efficiency (as well as comfort) requirements. It is supported by a mobile/desktop application setup 


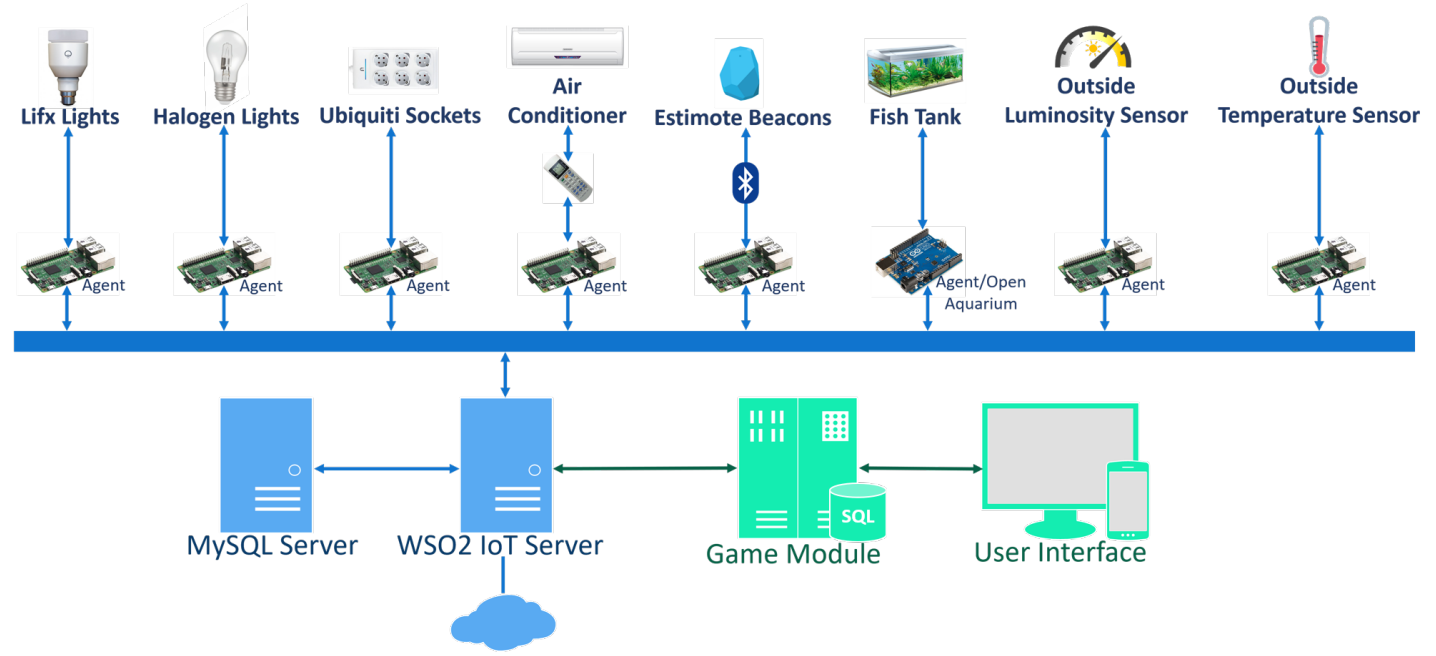

Fig. 3. Overview of the system architecture.

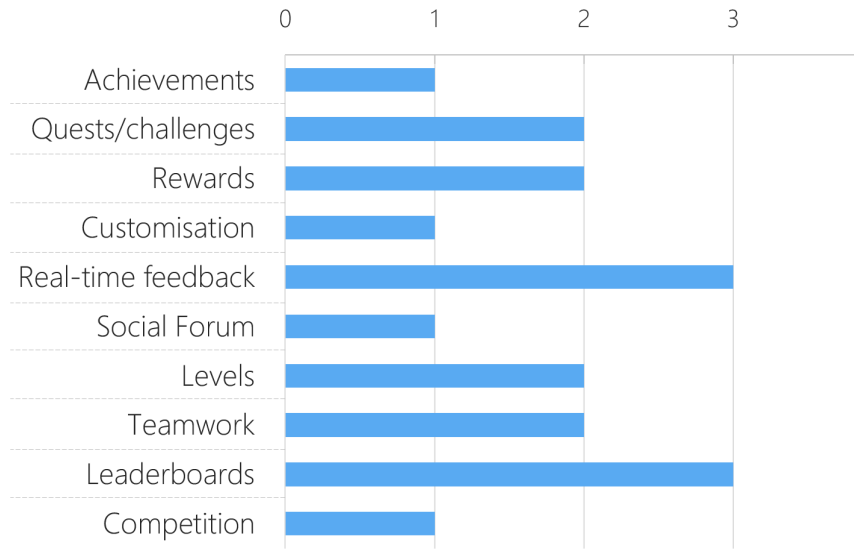

Fig. 4. Important game elements for the users.

which functions in co-operation with the room's sensors and actuators. The game is supported by a points system where the players' rewards depend on the support they provide to the system and on their eco-friendly behaviour. The achievement of stability in the system involving the player's motivation is expected. The system has to have the means to measure and deal with the evolution of gaming requirements.

There are two ways to win points. Players can complete challenges and tasks. Challenges are missions that the players urged to complete for a fixed period and can be an individual or team challenges. If the players meet the goals or significantly improve their statistics, then they will be rewarded. An example could be to improve energy efficiency by $5 \%$. Actions, such as opening the blinds in the open space, are tasks which the system, due to its retrofitting setup, cannot perform by itself. For example, when the need for opening the blinds is identified, the system requests the occupants signalling with blinking lights (prepared for identifying this type of request and calling the occupants attention). The occupant will then open the app to view more information about what is being
4 requested, and can then proceed to perform it and inform the system about its accomplishment. Every task and challenge has some points associated. If the challenge/task is completed, then the points are credited to the user. However, if the goal is not met, but there is a significant improvement, the participants win $10 \%$ of the challenge value. For example, if the players were challenged to increase savings by $10 \%$ but only achieved an improvement of $8 \%$. Players can also lose points, if they are present in the room and do not perform a requested task or if the energy efficiency drops to a specific configured value. This measure intends to prevent participation decay over time. Once the number of assignments is reduced, because the room has reached an optimised state, we need to ensure that the players are still engaged and trying to maintain good game results.

Players are ranked according to their points in the system, and the ranking is publicly available. Along the way, the players may also collect badges, a symbol of recognition for good practices and achievements.

The system presents some tips to help the user to manage energy consumption and collect all the badges efficiently. The game focuses on helping the players become aware and reduce their greenhouse gases emissions, and therefore helping the planet. We believe this aspect, combined with the game logic will provide the needed motivation to our players. The application includes visualisation interfaces, displaying the overall ranking and performance statistics. The occupant can also consult the global conditions of the room and set up the devices: the interface allows the players to monitor and control certain features of the system. Thus, the players can remain informed of their progress throughout the game. They can also observe the impact they cause in the game and make decisions based on this information.

\section{A. Evolution Of Gaming Requirements}

The main goal of this project is to manage energy consumption in an intelligent retrofitting environment efficiently. We want to verify if we can achieve this goal with humans being 
part of the system. We thus proposed Gamification as a nonfunctional requirement, which is used to keep the occupants engaged, challenged and supported throughout the experience. Given these requirements, the overall goals of the system and the positive feedback from the questionnaire, we developed a responsive gamified web application.

As proposed in game theory, the initial part of the game must be easy to accomplish, so that the occupants will feel comfortable using and interacting with it [14]. Over time, the game provides tougher challenges and achievements become more difficult. This evolution aims to motivate improved behaviour. This is a known situation in the evolution of requirements in gaming contexts. Moreover, when an optimised state in the CPS is reached, the number of assignments will reduce, and some players may start losing interest and lower their participation. At this time, the system is adjusted, and additional steps in the evolution of gaming requirements are addressed, to maintain the stability in the CPS system.

In this context, we have designed some preventive measures, depicted in Table I. Certain gamification features are not dependent on stabilisation (e.g. the performance statistics), which may discourage the players and therefore compromise elements that translate to important aspects of the occupants' behaviour in the CPS. Thus, we created specific adjustments, based on the feature in question, aiming to maintain engagement and flow in the system.

The CPS is used to check the evolution in the occupant's motivation and response. For instance, the occupant's presence in the system is confirmed through the use of the power sockets (consumption). On the other hand, the lack of motivation is determined when the system observes that the occupants fail to respond to the game requests. In this case, points are deducted, and badges are removed. Additional measures are being designed to motivate the players to go back to the game.

These measures, as well as the players' opinions, will be gathered to build requirements evolution measures. Building and maintaining motivation in the game is a crucial aspect here [14], [15].

We are currently designing evaluation measures for the existing developed solution. Specifically, users will respond to comfort evaluation questionnaires, providing feedback. Moreover, the data gathered, during specific periods, will be compared to identify the impact of our gamification strategy.

\section{B. Implementation}

The proposed solution has two major components, the cyber-physical system setup and the game logic.

The setup of the open space is detailed in [removed for blind review] and described in section sec:CStudy. The devices in the room are accessible through simple APIs and a WSO2 IoT $^{4}$ server platform, which is responsible for storing data in a MySQL database and changing the settings of the environment. This component enables the system to run on its own, without supervised control.

\footnotetext{
${ }^{4}$ Platform that enables management of IoT devices and mobiles for enterprises. https://wso2.com/iot
}

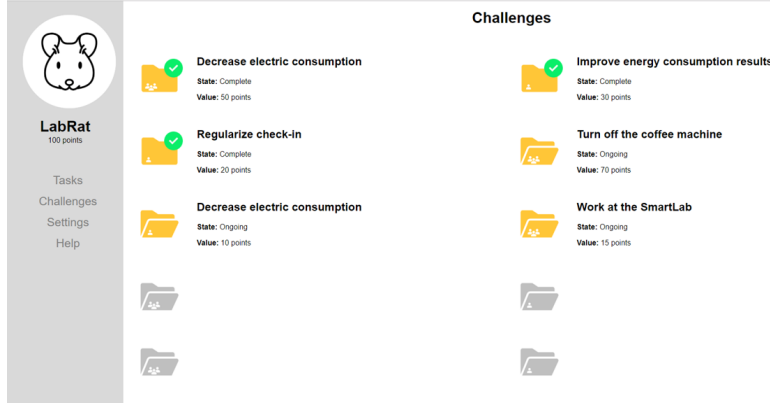

Fig. 5. Game application screen capture.

The game logic component (realised as a mobile/desktop app, see Figure 5) accommodates all the system's game logic, handle communication with the WSO2 IoT server and provide a user interface for the players. The users can interact with the system, define the system tasks to be realised by the Human (see Figure 6), and change the state of the environment through the use of a web application. The responsive user interface considers adaptability to different devices as well as user experience and usability issues.

Our two-part solution allows the system to function and keep efficiency levels when there are no players present in the room. The system can also be controlled directly by the player's.

\section{CONClusion And Future Work}

In this paper, we have introduced ongoing work regarding an office automation system that sees and interacts with the humans as system components to operate. Two sometimes conflicting variables are the core of the system behaviour to achieve stability: Comfort and Energy consumption. As humans interest tends to decrease with time, we propose to make use of Gamification to keep them engaged.

With our proposal we want to show how Gamification can help identify modifications in the system's requirements, given transformations in the behaviour of the occupants. Furthermore, we aim to demonstrate the influence of our adjustments in the engagement and motivation of the players. The system is currently built and running, and we are monitoring the effect of the Gamification in a long time frame to have significant results.

As future work, we foresee several interesting challenges. The first one is related to the privacy of the data. It is necessary to devise anonymity strategies for the data collected by the sensing devices and during the game. The second one is related to system security. We want to ensure that the system, including the different work-flows and system goals under various possible scenarios, are not vulnerable to attacks. Regarding the future, we are investigating other options to assess presence in the room, such as card reading sensors or triggered events to increase the accuracy of the data collecting process. 
TABLE I

RELATIONSHIP BETWEEN THE GAMIFICATION ELEMENTS AND THEIR IMPACT ON THE SYSTEM.

\begin{tabular}{l|l|l} 
Gamification feature & \multicolumn{1}{|c}{ Impact on CPS } & \multicolumn{1}{c}{ Future adjustment } \\
\hline Real-time feedback & Energy consumption & Informative messages \\
Rewards & Participation & Additional challenges \\
Challenges/Tasks & Energy consumption, participation, presence, teamwork & Introduction of penalties, new types of challenges \\
Ranking/Score & Presence, participation, energy consumption & Introduction of penalties \\
Badges & Energy consumption, participation, presence & Badge removal \\
Helpful tips & Energy consumption, participation & Trade points for tips
\end{tabular}

LabRats Hello admin!

\section{Create new task}

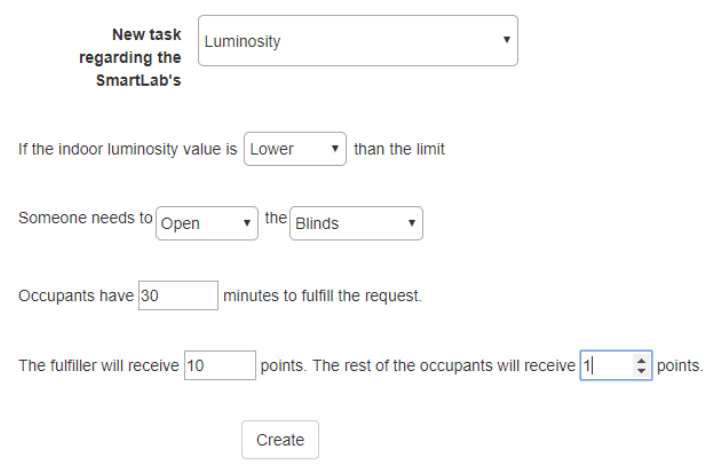

Fig. 6. Defining the tasks in the system.

\section{ACKNOWLEDGMENTS}

We thank NOVA LINCS Automated Software Engineering team and NOVA LINCS Research Laboratory (Ref. UID/CEC/04516/2013) for their financial support.

\section{REFERENCES}

[1] F. S. Nino, "The Science." [Online]. Available: http://www.un.org/climatechange/the-science/

[2] E. A. Lee and S. A. Seshia, Introduction to Embedded Systems: A Cyber-physical Systems Approach, 1st ed., ser. Electrical Engineering \& Computer Sciences. Lulu: LeeSeshia.org, 2012, oCLC: 915495730.

[3] W. Kastner, G. Neugschwandtner, S. Soucek, and H. M. Newman, "Communication systems for building automation and control," Proceedings of the IEEE, vol. 93, no. 6, pp. 1178-1203, Jun. 2005.

[4] "Hidden for double-blind review purposes."

[5] G. Schirner, D. Erdogmus, K. Chowdhury, and T. Padir, "The Future of Human-in-the-Loop Cyber-Physical Systems," Computer, vol. 46, no. 1, pp. 36-45, Jan. 2013.

[6] S. Deterding, M. Sicart, L. Nacke, K. O'Hara, and D. Dixon, "Gamification. Using Game-design Elements in Non-gaming Contexts," in CHI'11 Extended Abstracts on Human Factors in Computing Systems, ser. CHI EA '11. New York, NY, USA: ACM, 2011, pp. 24252428. [Online]. Available: http://doi.acm.org/10.1145/1979742.1979575

[7] K. Wiegers and J. Beatty, Software Requirements. Pearson Education, Aug. 2013, google-Books-ID: nbpCAwAAQBAJ.

[8] N. A. Ernst, J. Mylopoulos, and Y. Wang, "Requirements Evolution and What (Research) to Do about It," in Design Requirements Engineering: A Ten-Year Perspective, K. Lyytinen, P. Loucopoulos, J. Mylopoulos, and B. Robinson, Eds. Berlin, Heidelberg: Springer Berlin Heidelberg, 2009, pp. $186-214$.
[9] D. Sgouropoulos, E. Spyrou, G. Siantikos, and T. Giannakopoulos, "Counting and tracking people in a smart room: An IoT approach," in 2015 10th International Workshop on Semantic and Social Media Adaptation and Personalization (SMAP). IEEE, Nov. 2015.

[10] P. Lombriser, F. Dalpiaz, G. Lucassen, and S. Brinkkemper, "Gamified Requirements Engineering: Model and Experimentation," in Requirements Engineering: Foundation for Software Quality, M. Daneva and O. Pastor, Eds. Cham: Springer International Publishing, 2016, vol. 9619, pp. 171-187. [Online]. Available: http://link.springer.com/10.1007/978-3-319-30282-9_12

[11] B. Orland, N. Ram, D. Lang, K. Houser, N. Kling, and M. Coccia, "Saving energy in an office environment: A serious game intervention," Energy and Buildings, vol. 74, pp. 43-52, May 2014. [Online]. Available: http://linkinghub.elsevier.com/retrieve/pii/S0378778814000747

[12] "Open Aquarium." [Online]. Available: https://www.cookinghacks.com/documentation/tutorials/open-aquarium-aquaponics-fishtank-monitoring-arduino/

[13] S. Munir, J. A. Stankovic, C.-J. M. Liang, and S. Lin, "Cyber Physical System Challenges for Human-in-the-Loop Control." in Presented as part of the 8th International Workshop on Feedback Computing. San Jose, CA: USENIX, 2013.

[14] K. Werbach and D. Hunter, For the Win: How Game Thinking Can Revolutionize Your Business. Wharton Digital Press, 2012. [Online]. Available: https://books.google.pt/books?id=abg0SnK3XdMC

[15] S. Deterding, "Gamification: designing for motivation," interactions, vol. 19, no. 4, p. 14, Jul. 2012. [Online]. Available: http://dl.acm.org/citation.cfm?doid=2212877.2212883 